SHORT REPORT

\title{
Bilateral paramedian midbrain infarct: an uncommon variant of the "top of the basilar" syndrome
}

\section{K Spengos, J C Wohrle, G Tsivgoulis, G Stouraitis, K Vemmos, V Zis}

Occlusion of the rostral portion of the basilar artery can result in ischaemia of the midbrain and thalami, as well as of the temporal and occipital lobes. The so called "top of the basilar" syndrome manifests clinically as numerous combinations of abnormalities of alertness, sleep-wake cycle, and behaviour and oculomotor or pupillomotor functions. A 67 year old man presented with bilateral internuclear ophthalmoplegia, rubral tremor, and daytime somnolence. He was awake during the night and mostly agitated and aggressive. An ischaemic lesion was visible on the magnetic resonance (MR) image in the central portion of the midbrain just ventral to the aqueduct, clearly affecting the paramedian structures bilaterally. MR angiographic images demonstrated a hypoplastic basilar artery ending in both superior cerebellar arteries. Both posterior cerebral arteries were seen to arise from the corresponding internal carotid arteries via the posterior communicating branches. This unique case of an acute bilateral paramedian infarct represents a highly uncommon variant of the "top of the basilar" syndrome and was due to the affected ischaemic territory-that is, the "distal field" of the variant basilar artery.

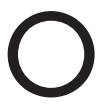
clusion of the rostral portion of the basilar artery can result in ischaemia of the midbrain and thalami, as well as of the temporal and occipital lobes. The so called "top of the basilar" syndrome is mostly embolic in origin and manifests clinically as numerous combinations of abnormalities including alertness, sleep-wake cycle, behaviour, and oculomotor or pupillomotor functions. ${ }^{12}$

Obstruction of the peduncular branches to the midbrain, which arise directly from the apex of the basilar artery, and the proximal portion of the posterior cerebral artery (PCA) supplying the paramedian periaqueductal structures can cause ipsilateral oculomotor nerve palsy or internuclear ophthalmoplegia (INO) as well as contralateral hemiparesis or hemiataxia with choreiform or tremor-like hyperkinesias (rubral tremor).

We report a unique case of an uncommon variant of the "top of the basilar" syndrome.

\section{CASE REPORT}

A healthy 67 year old man was working in his garden when he suddenly experienced dizziness and fell to the ground. Afterwards, he was slightly somnolent and unable to stand or articulate words. He was transferred to the nearest hospital, where he remained for two weeks in a general medical ward. A brain computed tomography (CT) scan was performed two days after symptom onset and demonstrated a small hypodense lesion in the central portion of the midbrain. The patient remained bedridden and somnolent during the period of hospitalisation. He mainly slept in the day and had nocturnal episodes of severe confusion and agitation, requiring treatment with haloperidol. He was diagnosed as having an ischaemic brainstem stroke. After discharge, the situation was complicated by fever; because he was unable to swallow, he could not be fed properly and developed aspiration pneumonia. The nocturnal episodes of aggression became more intense, and, during his efforts to stand up, he repeatedly fell to the ground sustaining several superficial injuries. The family physician suspected a recurrent stroke and referred him to our hospital for further evaluation.

On examination the patient was somnolent. However, he reacted to external stimuli to full wakefulness. He was then compliant and followed verbal orders but was severely dysarthric. Bilateral INO was manifest causing diplopia and dissociated nystagmus on horizontal gaze. Vertical gaze and convergence as well as pupillary functions were normal. No pyramidal signs or any motor or sensory deficit were present on clinical examination. He had bilateral ataxia with dysmetria in all four extremities, more pronounced on the left side. In addition, on voluntary movements or after emotional stress he presented impressive tremor-like hyperkinesias, especially in the upper extremities and the neck. He was unable to sit without direct body support. In the absence of continuous external stimulation, he fell asleep within a few moments. He was awake during the night and mostly agitated and aggressive, trying to hit or bite his caregivers.

After he was given antibiotics and fed through a nasogastric tube his general condition improved and diagnostic procedures, such as magnetic resonance imaging (MRI) could be undertaken. A hyperintense lesion of ischaemic origin was visible in the $\mathrm{T} 2$-weighted and the fluid attenuated inversion recovery (FLAIR) tomographic images in the central portion of the midbrain just ventral to the aqueduct, which was obviously affecting paramedian structures bilaterally, such as both the red nuclei and both the medial longitudinal fascicles, and presumably the reticular formation (fig $\mathrm{lC}-\mathrm{E}$ ). MR angiographic images in time-of-flight technique demonstrated low blood flow in the hypoplastic basilar artery, which seemed to end in both superior cerebellar arteries (fig 1A, B). Both PCAs were seen to arise from the corresponding internal carotid arteries via the posterior communicating branches.

After the patient had stabilised clinically, he was transferred to a rehabilitation centre. A slight improvement was reported at follow up three months later. He was able to sit and although the dysarthria persisted, it was less pronounced. His circadian sleep pattern had practically normalised and the episodes of nocturnal agitation had ceased. On the other hand, the bilateral INO remained unchanged.

\section{DISCUSSION}

"Top of the basilar" syndrome is known for the its variety of clinical manifestations. ${ }^{1}$ Our patient presented a unique

Abbreviations: INO, internuclear ophthalmoplegia; PCA, posterior cerebral artery 

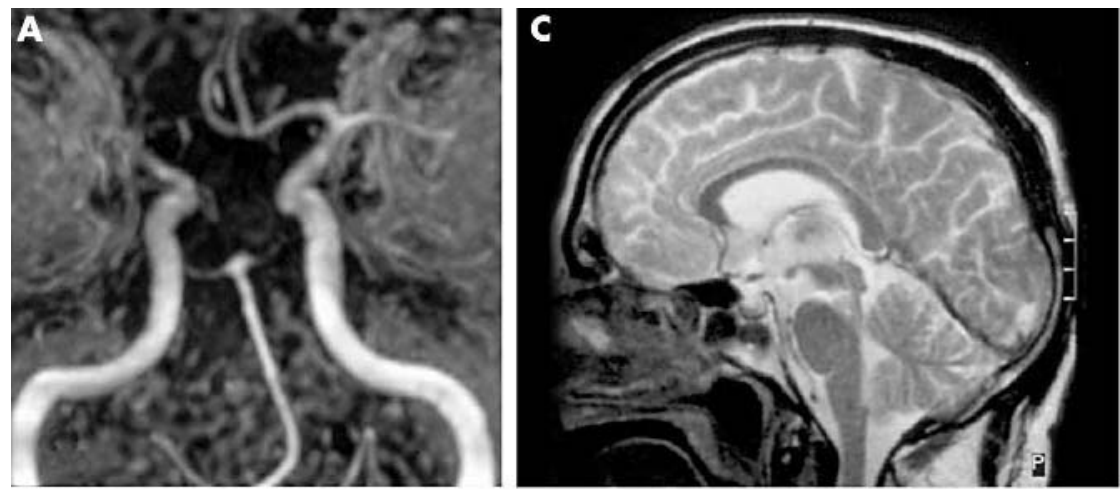

Figure 1 (A, B) Magnetic resonance angiographic images in time-of-flight technique demonstrating a hypoplastic basilar artery ending in both superior cerebellar arteries. Both posterior cerebral arteries are arising directly from the corresponding internal carotid arteries. A hyperintense lesion in the central portion of the midbrain just ventral to the aqueduct is visible in the sagittal (C) and axial T2-weighted (D) and FLAIR (E) magnetic resonance images suggesting an ischaemic nature of the underlying defect.
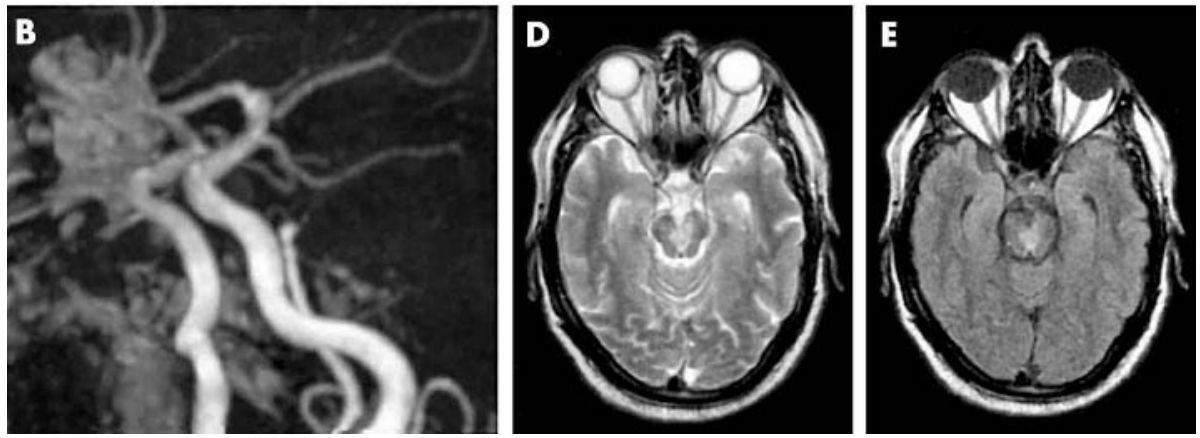

constellation of symptoms and signs due to an anatomical variation of the basilar system. To our knowledge, the combination of hypersomnolence and inversion of the sleep-wake cycle with bilateral INO, ataxia, and rubral tremor ${ }^{2}$ has not been reported previously. The combination has certain similarities with Benedikt's syndrome, as well as with Wernekinck's commissure syndrome, which is also characterised by bilateral tremor due to a bilateral lesion of the cerebellar peduncle. ${ }^{3}$

Bilateral INO is a very rare clinical feature of vertebrobasilar ischaemia. ${ }^{45}$ Bilateral ataxia can be attributed to a unilateral lesion of the brachium conjunctivum. Bilateral rubral tremor is a rarity and can be explained only by a paramedian bilateral lesion affecting both red nuclei. Such isolated butterfly-like midbrain infarcts with no thalamic or lobar involvement are extremely rare. ${ }^{6}$ There are only few descriptions of bilateral rostral mesencephalic periaqueductal lesions with prolonged hypersomnolence and third nerve palsies or INO. $^{7}$ Involvement of the reticular activating system and the lateral hypothalamus can cause sleep-wake cycle alterations. ${ }^{2}$

Our patient had an acute bilateral midbrain syndrome that was characterised by a sudden maximal neurological deficit at onset. The anatomical variation of both the PCAs arising from the anterior circulation explains why both thalami and the temporal and occipital lobes remained intact. It seems as if the median peduncular branches of the mesencephalic arteries, into which the basilar artery was draining, could have been occluded following embolism in the basilar artery apex. However, extensive diagnostic workup failed to identify any possible source of emboli. In the absence of other conventional risk factors (hypertension, diabetes mellitus, atrial fibrillation, coronary artery disease), the underlying cause of the infarct located in this highly vulnerable region could have been hypoperfusion of the paramedian midbrain territory. As demonstrated on MRI, the infarct affected paramedian structures bilaterally, namely the red nuclei, both medial longitudinal fascicles, and the reticular formation. This anatomical distribution can be regarded as the "distal field" of the variant basilar artery. The lesion corresponds to the bilateral occurrence of INO, rubral tremor and ataxia, and the disturbance of wakefulness.

Few similar clinical syndromes have been described with similarly located infarcts. Our patient had a brisk bilateral rubral tremor that, in combination with all other symptoms, represents a highly uncommon variation of the "top of the basilar" syndrome.

\section{Authors' affiliations}

K Spengos, G Tsivgoulis, G Stouraitis, V Zis, Department of Neurology, University of Athens, Athens, Greece

J C Wohrle, Department of Neurology, University of Heidelberg,

Universitätsklinikum Mannheim, Mannheim, Germany

K Vemmos, Department of Clinical Therapeutics, University of Athens, Athens, Greece

Competing interests: none declared

Correspondence to: Dr K Spengos, Department of Neurology, University of Athens, Vas. Sofias 82, 11528 Athens, Greece; spengos@ hol.gr

Received 18 July 2004

Revised version received 24 August 2004

Accepted 25 August 2004

\section{REFERENCES}

1 Caplan LR. "Top of the basilar" syndrome. Neurology 1980;30:72-9.

2 Mehler MF. The rostral basilar artery syndrome: diagnosis, etiology, prognosis. Neurology 1989;39:9-16.

3 Lhermitte F. Le syndrome cérebelleux: étude anatomo-clinique chez l'adulte. Rev Neurol 1958;98:435-77

4 Mehler MF. The neuro-ophthalmological spectrum of the rostral basilar artery syndrome. Arch Neurol 1988;45:966-71.

5 Finocchi C, Del Sette M, Croce R, et al. Bilateral ophthalmoplegia: an unusual sign of the top of the basilar artery syndrome. Ital J Neurol Sci 1996;17:301-4.

6 Fisher CM. The posterior cerebral artery syndrome. Can J Neurol Sci $1986 ; 13: 232-9$.

7 Facon E, Steriade M, Werthein N. Hypersomnie prolongée engendrée par les lésions bilatérales du système activateur médial: le syndrome thrombotique de la bifurcation du tronc basilaire. Rev Neurol 1958;98:117-33. 